
\title{
28 Research Suare \\ FASN Inhibition as a Potential Treatment for Endocrine-Resistant Breast Cancer
}

\section{Aleksandra Gruslova}

UT Health San Antonio: The University of Texas Health Science Center at San Antonio

\section{Bryan McClellan}

UT Austin: The University of Texas at Austin

\section{Henriette Balinda}

UT Health San Antonio: The University of Texas Health Science Center at San Antonio

\section{Suryavathi Viswanadhapalli}

UT Health San Antonio: The University of Texas Health Science Center at San Antonio

\section{Victoria Alers}

UT Health San Antonio: The University of Texas Health Science Center at San Antonio

\section{Gangadhara Sareddy}

UT Health San Antonio: The University of Texas Health Science Center at San Antonio

\section{Michael Garcia}

UT Health San Antonio: The University of Texas Health Science Center at San Antonio

\section{Linda deGraffenried}

UT Austin: The University of Texas at Austin

\section{Ratna Vadlamudi}

UT Health San Antonio: The University of Texas Health Science Center at San Antonio

Andrew J Brenner ( $\boldsymbol{D}$ brennera@uthscsa.edu )

UT Health San Antonio https://orcid.org/0000-0002-3985-253X

\section{Research Article}

Keywords: estrogen receptor, Fatty acid synthase

Posted Date: February 9th, 2021

DOl: https://doi.org/10.21203/rs.3.rs-173586/v1

License: (1) This work is licensed under a Creative Commons Attribution 4.0 International License. Read Full License 
Version of Record: A version of this preprint was published at Breast Cancer Research and Treatment on April 24th, 2021. See the published version at https://doi.org/10.1007/s10549-021-06231-6. 


\section{Abstract}

\section{Purpose}

The majority of breast cancers are estrogen receptor (ERa) positive making endocrine therapy a mainstay for these patients. Unfortunately, resistance to endocrine therapy is a common occurrence. Fatty acid synthase (FASN) is a key enzyme in lipid biosynthesis and its expression is commensurate with tumor grade and resistance to numerous therapies.

\section{Methods}

The effect of the FASN inhibitor TVB-3166 on ERa expression and cell growth was characterized in tamoxifen resistant cell lines, xenografts, and patient explants. Subcellular localization of ERa was assessed using a combination of immunofluorescence and subcellular fractionations. Palmitoylation and ubiquitination of ERa were assessed by immunoprecipitation. ERa and p-elF2a protein levels were analyzed by western blotting after treatment with TVB-3166 with or without the addition of palmitate or BAPTA.

\section{Results}

TVB-3166 treatment leads to a marked inhibition of proliferation in tamoxifen-resistant cells compared to the parental cells. Additionally, TVB-3166 significantly inhibited tamoxifen-resistant breast tumor growth in mice and decreased proliferation of primary tumor explants compared to untreated controls. FASN inhibition significantly reduced ERa levels most prominently in endocrine resistant cells and altered its subcellular localization. Furthermore, we showed that the reduction of ERa expression upon TVB-3166 treatment is mediated through the induction of endoplasmic reticulum stress.

\section{Conclusion}

Our preclinical data provide evidence that FASN inhibition by TVB-3166 presents a promising therapeutic strategy for treatment of endocrine-resistant breast cancer. Further clinical development of FASN inhibitors for endocrine resistant breast cancer should be considered.

\section{Introduction}

The past decade has seen significant advancement in increasing survival in the management of estrogen receptor alpha $(\mathrm{ERa})$ positive breast cancer. The use of selective estrogen receptor down-regulators and modulators (SERMs) (e.g., fulvestrant, tamoxifen) [1], mTOR inhibitors (e.g. everolimus) [2], aromatase inhibitors (Al) [3], and most recently the use of cyclin-dependent kinase inhibitors, (eg., ribociclib, Palbociclib,and abemaciclib [4-6]) have helped to extend the overall survival of the breast cancer patients. Yet, they do not offer an enduring cure and all patients will eventually succumb to their disease. The American Cancer Society predicts that over 42,000 women will die from breast cancer in 2020, with the majority expressing the estrogen receptor (ERa) [7]. Furthermore, the latest generation of targeted 
therapies has a higher level of toxicity relative to SERMs and Als. Thus, there is still a significant need to find additional treatment options that can help to eliminate the mortality associated with metastatic ER】 positive breast cancer, as well as to identify new therapies that are more effective, less toxic, and impact survival.

Alterations in endogenous lipid metabolism is an established hallmark of cancer and contributes to an aggressive and drug-resistant phenotype $[3,8,9]$. Among the enzymes responsible for lipid biosynthesis are the ATP citrate lyase (ACLY), acetyl-CoA carboxylase [9], stearoyl-CoA desaturase (SCD), and fatty acid synthase (FASN) [1, 9]. Briefly, endogenous synthesis of long-chain fatty acids, such as palmitate, is an NADPH dependent reaction that is mediated through FASN using acetyl-CoA and malonyl-CoA as substrates $[10,11]$. Intracellular palmitate serves as a substrate for both post-translation protein lipidation and membrane phospholipid biosynthesis, while also participating as an intracellular signaling molecule $[9,10]$. FASN was initially discovered as the oncogenic antigen-519 (OA-519) that was highly expressed in aggressive breast cancer [12]. Moreover, it was found that FASN inhibition using cerulenin, leads to an induction of apoptosis [12]. Since its discovery as an oncogenic antigen, numerous preclinical and clinical therapies have been developed to target its catalytic $\beta$-ketoacyl reductase and thioesterase domains $[10,13,14]$. However, early developed FASN inhibitors were limited to preclinical use due to unwarranted side effects. Newer and more targeted therapies have been developed with little to no side effects [8]. TVB-2640 is a potent and reversible inhibitor of the FASN enzyme that has been validated in multiple tumor cell lines, as well as in clinical studies $[13,15,16]$. TVB-2640 inhibits the ketoacyl reductase (KR) enzymatic activity of the FASN enzyme and is uncompetitive towards both NADPH and Acetoacetyl-CoA in inhibiting KR activity. The activity of TVB was seen across several cell lines, including MDA-MB-453, MDA-MB-231, MCF7, and BT-474. The pharmacokinetics [17] and metabolism of TVB-2640 have been examined with a series of in vitro and in vivo studies, including toxicokinetic studies. Overall, the PK properties determined to-date for TVB-2640 demonstrate that it is orally absorbed in humans and has a mean half-life of approximately 10-13 hours [17].

The safety and preliminary efficacy of TVB-2640 in a total of 78 patients with multiple primary tumors across 11 centers [13] was recently completed. Pharmacokinetic analyses showed a favorable profile with daily dosing, no attributable grade 3 adverse events at the recommended phase 2 dose of 100 $\mathrm{mg} / \mathrm{m} 2$, and excellent pharmacodynamics with rapid reduction in both palmitate production and a reciprocal increase in precursor malonyl carnitine in serum. Overall, 5 confirmed RECIST partial responses (cPR) and multiple patients with prolonged stable disease [18] ( $\geq 16$ weeks) were observed [19]. To preliminarily explore the activity of TVB-2640 in taxane resistant metastatic breast cancer, a dose expansion cohort at the recommended phase 2 dose was performed. Fifteen patients were enrolled, and represented a heavily pretreated population with an average of 7 prior regimens and all considered taxane resistant [19]. Interestingly, 2 of 3 patients with confirmed partial responses had tamoxifen refractory $E R \rrbracket+$ disease, as did all patients with stabilization beyond 24 weeks. While high FASN expression was relatively sensitive for predicting response, it was by no means specific as a significant percentage of patients with low FASN expression had significant stabilization of disease and included our longest responders. These findings suggested the possibility of selective activity of FASN inhibition in endocrine- 
resistant $E R \rrbracket+$ breast cancer that was not specifically dependent on the high expression of the enzyme [19]. Mechanistic studies show that FASN inhibition with TVB-3166, a TVB-2640 analog with a slightly lower molecular weight for in vitro use, in a tumor cell specific fashion disrupts lipid raft architecture, inhibits biological pathways such as lipid biosynthesis, PI3K-AKT-mTOR and $\beta$-catenin signal transduction, and inhibits expression of oncogenic effectors such as c-Myc [13]. Previous studies, including Menendez et al., have illustrated crosstalk between FASN and ERa, and that inhibition of FASN may lead to the reduction of ER囚 protein, induction of CDK inhibitors $\mathrm{p} 21^{\mathrm{CIP} 1}$ and $\mathrm{p} 27^{\mathrm{kip} 1}$, and suppression of estradiol stimulated proliferation and anchorage-independent colony formation [8] [20, 21]. Intriguingly, Zadra et al., observed a degradation of the androgen receptor (AR) in castration-resistant prostate cancer upon treatment with the FASN inhibitor, IPI-9119, that was mediated through endoplasmic reticulum stress (EnRS) [22, 23]. Both reduction in total AR protein and EnRS was ameliorated upon palmitate treatment [22], highlighting the role of FASN inhibition in induced EnRS. Though both preclinical and clinical studies have elucidated mechanisms of FASN inhibition for the treatment of multiple therapyresistant tumors, there is a lack of investigation on the potential for FASN inhibition as a therapy option for endocrine therapy resistant breast cancers.

Based upon our previous preliminary clinical data showing that FASN inhibition is beneficial in metastatic breast cancer patients, in this study, we demonstrate that inhibition of fatty acid synthase can improve outcomes in ER囚 positive, endocrine-resistant, metastatic breast cancer. Furthermore, we show that FASN inhibition specifically targets ERa through the induction of EnRS in tamoxifen-resistant breast cancer.

\section{Materials And Methods}

\section{Cell lines}

Human breast cancer cells MCF7 and ZR75 were purchased from the American Type Culture Collection (ATCC, Manassas, VA). Tamoxifen-resistant MCF7/TamR and ZR75-ESR537S mutant cells were described [24]. Each cell line was maintained according to the routine protocol and tested for mycoplasma using PCR Mycoplasma detection kit (PromoCell, Germany). Additionally, the cell lines were authenticated by STRF DNA profiling at UTHSA and UT Southwestern core facilities.

\section{Circulating Tumor Cells}

Circulating tumor cells were donated from a patient with a failed response to aromatase inhibitor therapy with ER囚 positive breast cancer. Isolated tumor cells were grown in short-term mammosphere culture prior to viability analysis. About $8 \mathrm{~mL}$ of whole blood from patients was collected in k2-EDTA (purple top), inverted $2 x$, and placed on ice as described previously with some modifications[25]. Red blood cells were lysed by adding Cell Dilution Buffer (v:v = 1:6) (ScreenCell, Westford, MA) and incubated at room temperature for 2 minutes. The majority of blood cells are removed using microfiltration (\#CY4FC-V2, ScreenCell). The identification of circulating tumor cells is carried out using immunofluorescence with anti-EpCAM-FITC (clone VU-1D9, StemCell Technologies, Cambridge, MA) and anti-CD45-PE (BD Bioscience). $C D 45-/ E p C A M+$ cell retained on microfilters are isolated individually into culture media. 


\section{In vitro proliferation assay}

For in vitro drug screening breast cancer cells were plated into 24-well plates in growth medium (DMEM containing $10 \%$ FBS and $1 \%$ L-glutamine). After overnight incubation cell plating medium was replaced with advanced MEM containing 1\% charcoal-stripped FBS, 1\% L-glutamine, and started treatment with TVB-3166 $(200 \mathrm{nM})$, tamoxifen $(1 \mu \mathrm{M})$ or combination for 14 days. All experiments were carried out using three biological replicates. Plates were incubated, imaged, and analyzed by an automated Live-Cell analysis system (IncuCyte). TVB-3166 was provided by Sagimet Biosciences (formerly 3V Biosciences).

\section{In vivo therapeutics studies in xenograft models}

All animal experiments were performed after obtaining UTHSA IACUC approval and using methods in the approved protocol. For xenograft tumor assays, tamoxifen-resistant MCF7/TamR cells $\left(5 \times 10^{6}\right)$ in $1: 1$ volume of Matrigel Matrix were injected into the mammary fat pads of 6-week-old SCID mice $(n=6$ per group). A 0.18 -mg sustained-release $17 \beta$-estradiol pellet was placed into the contralateral flank before injection. When tumors reach measurable size $\left(\sim 100-300 \mathrm{~mm}^{3}\right)$, animals were randomized into three treatment groups: TVB-3166 (60 mg/kg via oral gavage daily), tamoxifen ( $4 \mathrm{mg} / \mathrm{kg}$ via subcutaneous injection daily) or a combination. The body weights and tumor size were recorded three times a week for adverse toxic effects. Tumor volume was determined by measurements in two dimensions using calipers, with volumes defined as: $L \times W 2 / 2$, where $L$ is the longest dimension of the tumor and $W$ the shortest. At the end of the experiment, the mice were euthanized, and the tumors were removed, weighed, and processed for IHC staining.

\section{Patient-derived explants (PDEx) studies}

UT Health Hospital (San Antonio, TX) provided written consent allowing the use of discarded surgical samples for research purposes according to an institutional board-approved protocol (\# 20070684H). The protocol for an ex vivo culture model of breast tumors was adopted from published studies [24]. Briefly, de-identified fresh tumor samples were dissected into $1 \mathrm{~mm}^{3}$ specimens, and placed on hydrated gelatin sponges in explant media containing $5 \%$ CCS FBS at $37^{\circ} \mathrm{C}$. After $24 \mathrm{~h}$, media were replaced with new media ( $1 \%$ CCS FBS) containing vehicle or TVB $(200 \mathrm{nM})$ for $72 \mathrm{~h}$. Representative tissues were fixed in $10 \%$ formalin at $4^{\circ} \mathrm{C}$ overnight and subsequently processed into paraffin blocks. Sections were stained with hematoxylin and eosin and examined to confirm and quantify the presence/proportion of tumor cells. Immunohistochemistry was then performed.

\section{Immunohistochemistry (IHC)}

For xenograft and PDEx samples, $5 \mu \mathrm{m}$ sections were used for standard IHC protocol staining with ERa (1:50, Roche), Ki67 (1:100, Vector Laboratories Burlingame, CA) antibody in conjunction with proper protocol. A proliferative index was calculated as the percentage of $\mathrm{Ki}-67$ positive cells in five randomly selected microscopic fields at 40x per slide. For subcellular localization of ERa, cultured cells were fixed in $100 \%$ cold methanol and stained with ERa antibody (Abcam, \# ab16660) at 1:50 dilution. Goat-anti-rabbit IgG Alexa Fluor 488 (Abcam, \#ab150077) was used as the secondary antibody at 1:100 dilution. DAPI 
was used as a nuclear counterstain. PBS instead of the primary antibody was used as the secondary antibody only control.

\section{Western blot analysis and subcellular fractionation}

Cells were plated in T75 flask overnight in advanced MEM medium with $5 \%$ CS FBS and $1 \%$ glutamine. The following day medium was replaced for $1 \%$ CS FBS and TVB (200 nM) were added. The cells were treated for 24 or 72 hours. Cells were lysed in RIPA buffer containing protein inhibitors. A total of $20 \mu \mathrm{g}$ protein was resolved in 12\% SDS polyacrylamide gel, transferred onto a PVDF or nitrocellulose membrane and blocked by $5 \%$ non-fat milk or BSA (Bovine Serum Albumin) in TBS-T buffer for $1 \mathrm{~h}$ at room temperature. Membrane was probed overnight at $4^{\circ} \mathrm{C}$ with primary antibody followed by $1 \mathrm{~h}$ incubation with secondary antibody at room temperature. Signal detection was done using ECL chemiluminescent kit (Invitrogen, \#WP20005) or West Femto Maximum Sensitivity Substrate (Thermo Scientific, \#34095) with the membranes visualized on a Kodak Image Station or Syngene Imager with GeneSnap software. Primary antibodies used were GAPDH (1:1000), ERa_SP1 (1:500), p-elF2a (1:1000, CST, \#9727), elF2a (1:1000, CST \#9722), ERa (1:1000, CST \#8644T), and secondary antibodies used were anti-mouse-HRP (1:2000) and anti-rabbit-HRP (1:3000). Subcellular fractionation was performed using a Subcellular Protein Fractionation kit (Thermo Scientific), according to the manufacturer's instructions.

\section{Palmitate-BSA Conjugation and Cell Treatments}

Sodium Palmitate (Millipore Sigma, \#P9767) was heated to $70^{\circ} \mathrm{C}$ for 30 minutes in autoclaved $\mathrm{H}_{2} \mathrm{O}$. Aqueous solution of sodium palmitate was added dropwise to free fatty acid (FFA)- stripped BSA solution and heated at $37^{\circ} \mathrm{C}$ for 2 hours to finalize conjugation to a 6:1 molar ratio of palmitate to BSA. Prior to cell treatment stock solutions of either FFA stripped BSA (vehicle control) or Palmitate: BSA (6:1 molar ratio) were heated to $370 \mathrm{C}$ and added to $2 \%$ charcoal-stripped FBS media. MCF7 and MCF7/TamR cells were seeded in six-well plates at a density of 350,000/well and allowed to adhere for 24 hours. $10 \%$ FBS DMEM media was replaced with $2 \%$ charcoal-stripped FBS media containing either BSA, Palmitate:BSA, TVB-3166, TVB-3166 + BSA, or TVB-3166 + Palmitate:BSA. Additionally, cells were pretreated with 1,2Bis(2-Aminophenoxy) ethane-N,N,N',N'-tetraacetic acid (BAPTA) (Millipore Sigma Aldrich, \#A4926) for 60 minutes $(20 \mu \mathrm{M})$ prior to the addition of TVB-3166 (200 nM) for 72 hours.

\section{ERa palmitoylation}

Detection of ERa palmitoylation was performed using Acyl-Biotin Exchange (ABE) assay as previously described [26]. Briefly, breast tumor cells were cultured with or without TVB-3166 (200 nM) then lysed in ice-cold lysis buffer containing $50 \mathrm{mM} \mathrm{N}$-ethylmaleimide. BSA assay was used to quantify the protein and $500 \mu \mathrm{g}$ of protein was subjected to immunoprecipitation with protein $\mathrm{G}$ sepharose beads (Millipore Sigma) using 1-5 mg of antibody against ERa (Abcam). Each sample of beads was split into two and incubated with or without hydroxylamine (HAM). Selective labeling of palmitoylated cysteine was performed using the buffer containing $3 \mu \mathrm{M}$ Biotin-BMCC (ProteoChem). The samples were boiled at $80^{\circ} \mathrm{C}$ for 10 minutes then resolved on SDS-PAGE gels. Streptavidin-HRP (Invitrogen) at 1:5000 dilution was used for detection of palmitoylated proteins. 


\section{RNA sequencing}

RNA-seq was performed using the UTHSA core-established protocol. Briefly, patient-derived explants were treated with either vehicle or TVB $(200 \mathrm{nM})$ for $72 \mathrm{~h}$, and total RNA was isolated using RNeasy mini kit (Qiagen) according to the manufacturer's protocol instructions. NGS libraries were prepared from total RNA using Illumina TruSeq stranded RNA Sample Prep kit following manufacturer's instructions and sequenced with $50 \mathrm{bp}$ single-read module using Illumina HiSeq 3000 system. After adapter trimming and demultiplexing, short-read sequence reads were processed using TopHat2 and HTSeq for genome alignment and expression quantification. Differential expression analysis was performed by DEseq and significant genes with at least 1.5 -fold change with $p<0.01$ were chosen for analysis. The interpretation of biological pathways using RNA-seq data was performed using gene set enrichment analysis. RNA-seq data have been deposited in the GEO database under accession number

\section{Human Phospho-Receptor Tyrosine Kinase Array}

MCF7/TamR cells were treated with either Tamoxifen (Tam, 1 mM), TVB-3166 (200 nM), or a combination for 72 hours, then relative levels of RTK-phosphorylation were determined using the Proteome Profiler Human Phospho-RTK Array kit following the manufacturer's protocol (R\&D Systems). The phospho-RTK array membranes were imaged using a Licor Fc imager with chemiluminescence. Analysis was performed using Licor's Studio Imager Lite, where background corrected mean pixel density is depicted as signal.

\section{Statistics}

Data represented in the bar graphs are shown as mean \pm SE. T-test was performed for all comparisons. Phospho-RTK array data was analyzed using Prism 8 software. A value of $p<0.05$ was considered as statistically significant. RNA-seq data were analyzed using IPA software.

\section{Results}

Inhibition of FASN blocks cell growth and cell cycle progression in tamoxifen resistant breast cancer cells through reduced ERa expression

The effects of FASN inhibition on the growth of tumor cells has been confirmed in several breast cancer cell lines including ER + cell lines MCF7 and ZR75, and triple-negative MDA-MB-231 cells. To ascertain whether FASN inhibition can inhibit growth in endocrine-resistant cells, we evaluated the proliferation rate of both parental MCF7 and their derivative from long term exposure to Tamoxifen (MCF7/TamR). Cells were cultured with either Tamoxifen (Tam, $1 \mu \mathrm{M})$, TVB-3166 (200 nM), or the combination. TVB-3166 is the preclinical version of TVB-2640 with a slightly lower molecular weight and increased solubility compared to TVB-2640 [13]. TVB-3166 led to a significant growth inhibition in both MCF-7 and MCF/TamR cell lines (Fig. 1a). Notably, TVB-1366 inhibited cell growth by 50\% in MCF7/TamR cells as compared to $20 \%$ in the parental MCF7 cells. Moreover, when TVB-3166 was combined with Tam, the combination resulted in complete growth inhibition of both Tam-sensitive and Tam-resistant MCF7 cells. 
This was associated with flow cytometry cell cycle analysis with propidium iodide DNA staining. A substantial increase of $S$ phase population at 72 hours is suggestive of DNA-damage or replicative stress (Fig. 1b). Western blot analysis of ER囚 protein levels showed a time-dependent loss of protein expression after TVB-3166 treatment in MCF7 and MCF7/TamR cells, which was markedly greater in MCF7/TamR cells (Fig. 1c). Following $72 \mathrm{~h}$ of exposure protein levels decreased by $90 \%$ in the MCF7/TamR cells, while only $20 \%$ in the parental cells. To evaluate the association of FASN inhibition with ERa we tested the effect of TVB-3166 on breast cancer cells with mutant estrogen receptor alpha gene (ESR1). In particular, TVB-3166 significantly inhibited ERa expression in ZR75-mutY537S cells compared to the control (Fig. 1d). ERa protein decreased by $70 \%$ and $90 \%$ after 24 and 72 hours, respectively. Using circulating tumor cells (CTCs), we evaluated the effect of FASN inhibition on primary tumor cultures. CTCs were collected from a patient with ER + disease failing aromatase inhibitor treatment and established into a short term mammosphere culture. (Fig. 1e) shows that after 72 hours of TVB exposure (200 nM) there was a $50 \%$ decrease in cell viability $(p<0.05)$, confirming our in vitro results. This data supports that FASN inhibition with TVB-3166 is associated with ERa signaling pathways.

\section{TVB-3166 inhibits growth of tamoxifen resistant breast tumors in mice}

In vivo efficacy of FASN inhibition on breast tumor growth was investigated in MCF7/TamR cells. Upon noticeable tumor size (100-300 $\mathrm{mm}^{2}$ ), nude mice bearing MCF7/TamR cells were randomized in 3 treatment groups: tamoxifen (100 $\mu \mathrm{g} /$ mice, subcutaneous), TVB-3166 (60 mg/kg, oral gavage) or combination. Eight weeks of treatment with TVB alone or in combination with tamoxifen resulted in statistically significant inhibition of MCF7 tamoxifen-resistant xenografts growth ( $\mathrm{n}=6$ mice/group) compared to tamoxifen alone (Fig. 2a). As shown in (Fig. 2b), the average size of the tumors in the combination group was $50 \%$ and $30 \%$ smaller compared with tamoxifen or TVB groups, respectively. Animals tolerated the treatment very well without any effect on their weight. These data indicate that TVB-3166 is a potent inhibitor of the growth of endocrine-resistant breast tumors in vivo with no overt signs of toxicity in mice. Furthermore, immunohistochemistry analysis of tumors showed significant decrease of ERa levels after TVB treatment alone or in combination with tamoxifen $(p=0.002$ and $p=$ 0.003 , respectively) (Fig. 2c and $2 d$ ).

\section{TVB-3166 inhibits proliferation in patient derived primary tumor explants}

Given potential differences between in vitro and in vivo we used an ex vivo culture model of primary breast tumors to characterize changes associated with FASN inhibition. This model maintains the native tissue architecture and critical cell-to-cell signaling of the tumor microenvironment and thus better recapitulates the complexity and heterogeneity of human breast cancer in a laboratory setting.

Additionally, these ex vivo techniques allow quantification of clinically relevant endpoints that are more applicable to patient treatment than established cell lines. In brief, fresh tumor tissue was obtained from 
surgical resection or biopsy, dissected into $1 \mathrm{~mm}^{3}$ specimens, and placed on hydrated gelatin sponges in explant media at $37^{\circ} \mathrm{C}$. After 24 hours, new media containing vehicle or investigational agents was added (Fig. 3a). Four ER+, HER2- breast tumor specimens taken from treatment naïve patients were cultured in the absence or presence of TVB-3166 (200 nM) for 72h. Immunohistochemistry analysis indicated a decrease in proliferation after TVB treatment. Ki67 staining was significantly lower in treated tumors compared to untreated ( $14 \%$ vs $36 \%, p<0.001)$ (Fig. 3b). Furthermore, FASN inhibition leads to a significant reduction in the ER囚 levels in tumors $(p<0.01)$ (Fig. $3 c)$.

\section{Identification of differentially expressed genes in response to TVB-3166 treatment}

To investigate specific biological outcomes from treatment in tamoxifen-resistant breast cancer, RNA sequencing, and phospho-receptor tyrosine kinase (RTK) array were performed in response to treatment with TVB-3166, tamoxifen, or in combination. RNA sequencing analyses revealed that TVB significantly altered the mRNA expression of 219 genes $(p<0.01)$ in primary patient-derived breast tumor explants compared to vehicle control $(n=2)$ (Fig. 4a). Gene set enrichment analysis (GSEA) of the differentially expressed genes ranked by fold-change showed 2 enriched gene sets: 1) upregulation of genes downregulated in ESR1 positive breast tumors compared to the ESR1 negative tumors (Fig. 4b.); and 2) downregulation of genes involved in invasiveness signature resulting from cancer cell-microenvironment interaction (Fig. 4c.). Additionally, the phosphorylated-RTK assay in MCF7 and MCF7/TamR cells revealed eight altered $(p<0.01)$ phosphorylation levels of RTKs affected in response to a combination of TVB3166 and tamoxifen compared to tamoxifen alone. Of the eight, the most dramatic changes were in the EGFR, FGFR2a, Tie2, and ROR2 phosphorylation. TVB-3166 and tamoxifen combination treatment increased the phosphorylation of ROR2 and EGFR dramatically compared to treatment with tamoxifen alone (Fig. 4d).

TVB-3166-induced ERa degradation in endocrine resistant breast cancer is mediated through enhanced endoplasmic reticulum stress.

ERa is a nuclear ligand-activated transcription factor and also an extrinsic plasma membrane receptor. ERa resides predominantly in the nucleus, while unbound ERa shuttles between the cytoplasm and nucleus. To confirm that long-term tamoxifen treatment causes translocation of ERa out of the nucleus to the cytoplasm [27], we extracted ERa from three different cell fractions (cytoplasm, membrane and nucleus) of MCF7/TamR cells and its parental MCF7 cell line. Western blot data showed that in MCF7 cells, more than $50 \%$ ERa was in the nucleus, whereas in MCF7/TamR cells, ERa level in cytoplasm was significantly higher compared to the parental cell line $(p=0.025)$ (Fig. 5a). Moreover, there was an appreciable degradation of total ERa protein in MCF7/TamR cells in response to TVB and tamoxifen combination treatment (Fig. 5b). In order to understand the localization and levels of ERa in response to TVB-3166 treatment, we investigated the levels of palmitoylation and ubiquitination of the ERa in MCF7/TamR cells. However, we observed no major differences in TVB-3166- treated and untreated samples in the levels of palmitoylated and ubiquitinated ERa protein (Fig. 1S). We also investigated the 
involvement of lysosomes in TVB-3166-dependent ERa degradation by pre-treating the cells with chloroquine, a drug that inhibits lysosomal enzymes by changing endosomes and lysosomes internal $\mathrm{pH}$ [28]. Our data showed that there was increased lysosomal degradation in the presence of TVB-3166, which was unexpected (Fig. 2S).

Alterations in membrane lipid composition are known to result in the induction of EnRS [29] (Fig. 6a). In periods of EnRS, the protein kinase $R$ (PKR)- like endoplasmic reticulum kinase (PERK) is activated resulting in serine phosphorylation of the eukaryotic initiation factor-2 complex in the alpha subunit (elF2a) [30]. This phosphorylation results in a competitive inhibition of the guanine nucleotide exchange factor for its beta subunit (elF2 $\beta$ ), ultimately leading to an inhibition of protein translation [31]. Additionally, previous studies have elucidated the role of FASN inhibition leading to EnRS in prostate cancer that was attenuated upon treatment with palmitate, the major product of FASN [22]. To investigate the effects of FASN inhibition and EnRS induction in tamoxifen-resistant breast cancer, both MCF7/TamR and MCF7 cells were subjected to treatment with or without $200 \mathrm{nM}$ of TVB in combination with either palmitate or BSA. Palmitate was conjugated to BSA in a 6:1 molar ratio, to be transported into the cell. Treatment with TVB-3166 resulted in a decrease in total ERa protein that was only apparent in MCF7/TamR cells. Additionally, the decrease in ERa expression was accompanied by an increase in EnRS, as indicated by levels of phosphorylated-elF2a (p-elF2a) (Fig. 6b). Moreover, the addition of palmitate rescued the expression of ERa, while ameliorating markers of EnRS (p-elF2a) (Fig. 6b). The quantification of band intensity revealed a statistically significant $(p=0.02)$ increase in ERa protein and decrease in $p$-elF2a $(p \leq .05)$, upon the addition of palmitate when treated with TVB-3166 compared to TVB-3166 alone (Fig. 6b). Considering the addition of palmitate attenuated stress in response to FASN inhibition, we sought to determine if the use of an EnRS inhibitor would have similar effects upon treatment with TVB-3166. Intriguingly, pretreating with the EnRS inhibitor, BAPTA, prior to the addition of TVB-3166 for 72 hours rescued ERa expression while lowering EnSR (p-elF2a) similar to that of the TVB + palmitate treatment (Fig. 6c). This rescuing effect of ERa along with the decrease in EnRS was only demonstrated in tamoxifen-resistant cells and not tamoxifen sensitive, indicating a cell type specific response.

\section{Discussion}

Estrogen positive breast cancer accounts for nearly $70 \%$ of breast cancer-related deaths, and often is treated with estrogen receptor antagonists such as tamoxifen. Unfortunately, ERa targeted therapies can result in resistance, subsequent metastasis, and death. Reprogramming of lipid metabolism is an established hallmark of cancer and is associated with drug-resistance, including resistance to antiendocrine therapies. The rate-limiting enzyme for endogenous lipid biosynthesis, fatty acid synthase (FASN), is overexpressed in numerous cancers and is associated with drug-resistance. The FASN inhibitor TVB-2640 has shown preliminary evidence of activity in a phase 1 clinical trial dose expansion arm for metastatic breast cancer. In this study, we illustrate that FASN inhibitor, TVB-3166 (preclinical version of TVB-2640), significantly reduces growth and proliferation of tamoxifen resistant breast cancer in in vitro, ex-vivo and in vivo models by inducing ERa degradation through increased endoplasmic reticulum stress. 
The inhibition of FASN has several consequences that result in either apoptosis or stasis in cell progression [13]. The primary product of FASN includes the long-chain fatty acid, palmitate, that can posttranslationally modify oncoproteins, such as Wnt and epidermal growth factor receptor (EGFR) that facilitate their intracellular localization and membrane hydrophobicity [32]. Moreover, palmitate itself undergoes subsequent alterations to generate phospholipids for membrane synthesis, which is essential for rapidly proliferating tissue [33]. A phase 1 study demonstrated a decrease in circulating palmitate metabolites in patients treated with TVB-2640 [19]. FASN is transcriptionally and post-transcriptionally regulated through $\mathrm{PI} 3 \mathrm{~K} / \mathrm{Akt} / \mathrm{mTOR}$ pathway downstream of receptor tyrosine kinases (RTKs), such as EGFR [34]. Thus, FASN-induced palmitoylation of RTKs could mediate a feedforward loop to support lipid metabolic programming in anti-endocrine resistance. Additionally, we have also observed an increase in malonyl-carnitine, a more stable derivative of the FASN substrate malonyl-CoA [19]. Malonyl-CoA has a short half-life and is therefore difficult to measure. Thus, the decreased cellular pool of palmitate for membrane synthesis and post-translational modifications could explain the growth inhibitory effects of FASN inhibition observed.

In response to treatment with tamoxifen, TVB-3166, or in combination, RNA sequencing and phosphoreceptor tyrosine kinase arrays demonstrated a significant increase in the receptor tyrosine kinase-like orphan receptor-2 (ROR2) when treated with the combination of TVB and tamoxifen compared to tamoxifen treatment alone in tamoxifen-resistant cells. Classically, both ROR1 and ROR2, are involved in neurogenesis and embryonic development. Moreover, expression of ROR1 and ROR2 is associated with increased invasion and poor prognosis in numerous cancers [35,36]. Interestingly, in certain cancers, ROR2 expression exhibits tumor-suppressing characteristics. Recent studies have illustrated ROR2 hypermethylation in nasopharyngeal, esophageal, and breast cancer cell lines promoted EMT and proliferation [35]. Additionally, ROR2 overexpression resulted in reduced Akt and $\beta$-catenin signaling in multiple cancer cell lines [35]. Not only does ROR2 possess tumor-suppressing traits but is also implicated in inducing apoptosis through the EnRS pathway. Recently, ROR2 promoted apoptosis in ovarian carcinoma through the induction EnRS sensing protein, inositol requiring enzyme-1a (IRE1a) and downstream C/EBP homologous protein (CHOP) [35, 36]. Thus, ROR2 overexpression observed in response to TVB and tamoxifen treatment could be illustrating tumor-suppressing characteristics.

Additionally, we demonstrate that treatment with TVB-3166 leads to a decrease in ERa protein that was specific to tamoxifen-resistant breast cancer compared to the tamoxifen sensitive. Several studies have elucidated an increase in cap-dependent mRNA translation through mTOR and ERK pathways as a mechanism of acquired tamoxifen resistance. Moreover, FASN inhibition has been demonstrated to attenuate Akt and mTORC1 signaling in multiple studies. In our lab, we observed an inhibition in the phosphorylation of Akt, which is upstream of mTORC1. Pathways upregulated in tamoxifen resistance, such as mTOR, involve the phosphorylation of translational inhibitory complexes such as $4 \mathrm{E}$ binding proteins (4E-BP1) leading to an alleviation of elF4E cap-binding protein. The mRNA translational initiation complex is composed of several elF4F family members that include an RNA helicase (elF4A), scaffolding protein (elF4G), and the rate-limiting cap-binding protein (elF4E) [30,31]. Moreover, many oncoproteins and growth factors such as cyclin D1 and vascular endothelial growth factor (VEGF) are encoded by 
mRNAs with longer than average 5 ' untranslated regions (UTRs) [30]. Translational complexes, such as eIF4E, have an enhanced affinity for longer UTRs. More specifically, using a mutated elF4E that renders it unable to become phosphorylated resulted in an attenuation in proliferation in response to tamoxifen in tamoxifen-resistant cell lines [31]. This upregulation in mRNA cap-dependent translation pathways in tamoxifen- resistance holds the premise for an increased sensitivity to FASN inhibition in tamoxifenresistant compared to tamoxifen sensitive breast cancer.

We observed that FASN inhibition induced ERa degradation is mediated through the EnRS pathway. Historically, higher ratios of phosphatidylcholine to phosphoethanolamine as well as increases in intracellular cholesterol lead to EnRS [23]. Studies have shown alterations in intracellular membrane lipids as well as a compensatory increase in cholesterol biosynthesis in response to FASN inhibition. Recent studies, conducted by Zadra et al., 2019, illustrated that FASN inhibition in prostate cancer leads to an alteration in phospholipids within the EnR membrane [22]. The initiation of protein translation involves both the recognition of the start codon in mRNA by the preinitiation complex as well as the recruitment of various eukaryotic initiation factors (elFs) [31]. elFs 1, 1A, 2, 3, 4 and 5 are all involved in translation[31]. Moreover, the elF2 complex is the site of translational control in periods of starvation, stress, or viral infection[31, 37]. EnRS through the PKR like endoplasmic reticulum kinase (PERK) results in the phosphorylation of eukaryotic initiation factor $2 a$ [23]. This results in protein translation inhibition, which could explain the decrease in ERa protein levels observed upon FASN inhibition. This EnRS response to FASN inhibition was only observed in tamoxifen-resistant breast cancer; however, the specific response could be due to the increased reliance upon translational machinery that has been recorded previously in acquired tamoxifen resistance [30, 31]. Intriguingly, the increased expression of ROR2 recorded in the phospho-RTK assay could be a potential mediator of endoplasmic reticulum stress. Previous studies connecting ROR2 expression to CHOP induced apoptosis highlight a potential connection to the PERK/p-eIF2a EnRS pathway [36]. Not only does p-elF2a lead to a decrease in protein translation, but also allows the initiation complex to continue down the mRNA until it reaches the alternate reading frame [31]. The alternate reading frame of mRNA is the location of translation for EnRS response proteins, such as CHOP and ATF-4 [30, 31]. Thus, the overexpression of ROR2 from treatment with TVB and tamoxifen could potentially be contributing to endoplasmic reticulum stress.

We previously preliminarily demonstrated in a phase 1 study that FASN inhibition is a viable treatment option for ER + breast cancer patients that have become resistant to anti-endocrine therapies [19]. In this study, we explore the potential mechanism and have shown that FASN inhibitor, TVB-3166, targets ERa protein for degradation in tamoxifen-resistant breast cancer. This loss in ERa protein was found to be as a result of increased endoplasmic reticulum stress. Future studies should investigate the connection to lipid metabolic programming and the mRNA translational network. Moreover, more specific mechanisms linking FASN expression directly to acquired anti-estrogen resistance should be conducted. Finally, more investigation should be done illustrating the role of ROR2 in tamoxifen-resistant breast cancer. With more targeted and less toxic therapy options, patients are extending not only their life, but their quality of life. FASN inhibition, either as a monotherapy or in combination, could be a potential novel treatment for antiendocrine therapy-resistant breast cancer.

Page 13/23 


\section{References}

1. Menendez J, Lupu R (2017) Fatty acid synthase regulates estrogen receptor-a signaling in breast cancer cells. Oncogenesis 6(2):e299-e299

2. Robertson JF et al (2016) Fulvestrant $500 \mathrm{mg}$ versus anastrozole $1 \mathrm{mg}$ for hormone receptor-positive advanced breast cancer (FALCON): an international, randomised, double-blind, phase 3 trial. The Lancet 388(10063):2997-3005

3. Lupu R, Menendez JA (2006) Targeting fatty acid synthase in breast and endometrial cancer: An alternative to selective estrogen receptor modulators? Endocrinology 147(9):4056-4066

4. Dickler MN et al (2017) MONARCH 1, a phase Il study of abemaciclib, a CDK4 and CDK6 inhibitor, as a single agent, in patients with refractory HR+/HER2 - metastatic breast cancer. Clin Cancer Res 23(17):5218-5224

5. Hortobagyi GN et al (2016) Ribociclib as First-Line Therapy for HR-Positive, Advanced Breast Cancer. N Engl J Med 375(18):1738-1748

6. De Angelis $\mathrm{C}$, Ignatiadis M, Piccart-Gebhart M, Cyclin-dependent kinases 4 and 6 inhibitors (CDK4/6i) versus chemotherapy in luminal B early breast cancer: lessons from the CORALLEEN trial. Annals of Translational Medicine, 2020. 8(21)

7. Desantis CE et al., Breast cancer statistics, 2019. CA: A Cancer Journal for Clinicians, 2019. 69(6): p. $438-451$

8. Menendez JA, Lupu R (2017) Fatty acid synthase regulates estrogen receptor-a signaling in breast cancer cells. Oncogenesis 6(2):e299-e299

9. Jones SF, Infante JR (2015) Molecular pathways: fatty acid synthase. Clin Cancer Res 21(24):54345438

10. Menendez JA, Lupu R (2007) Fatty acid synthase and the lipogenic phenotype in cancer pathogenesis. Nat Rev Cancer 7(10):763-777

11. Corominas-Faja B et al (2017) Clinical and therapeutic relevance of the metabolic oncogene fatty acid synthase in HER2 + breast cancer. Histology histopathology 32(7):687

12. Kuhajda FP et al., Fatty acid synthesis: a potential selective target for antineoplastic therapy. Proceedings of the National Academy of Sciences, 1994. 91(14): p. 6379-6383

13. Ventura R et al (2015) Inhibition of de novo palmitate synthesis by fatty acid synthase induces apoptosis in tumor cells by remodeling cell membranes, inhibiting signaling pathways, and reprogramming gene expression. EBioMedicine 2(8):808-824

14. Patel M et al (2015) Abstract CT203: Report of a first-in-human study of the first-in-class fatty acid synthase (FASN) inhibitor TVB-2640. Can Res 75(15 Supplement):CT203-CT203

15. Heuer TS et al., FASN inhibition and taxane treatment combine to enhance anti-tumor efficacy in diverse xenograft tumor models through disruption of tubulin palmitoylation and microtubule organization and FASN inhibition-mediated effects on oncogenic signaling and gene expression. 2016 
16. Brenner A et al., First-in-human study of the first-in-class fatty acid synthase (FASN) inhibitor, TVB2640 as monotherapy or in combination-final results of dose escalation. 2015

17. Falchook $\mathrm{G}$ et al., Abstract CT153: First in human study of the first-in-class fatty acid synthase (FASN) inhibitor TVB-2640. 2017, AACR

18. WäsTER $P$ et al (2011) Ultraviolet exposure of melanoma cells induces fibroblast activation protein-a in fibroblasts: Implications for melanoma invasion. Int J Oncol 39(1):193-202

19. Brenner A et al., Abstract P6-11-09: Heavily pre-treated breast cancer patients show promising responses in the first in human study of the first-In-class fatty acid synthase (FASN) inhibitor, TVB2640 in combination with paclitaxel. 2017, AACR

20. Cariou S et al., Down-regulation of p21WAF1/CIP1 or p27Kip1 abrogates antiestrogen-mediated cell cycle arrest in human breast cancer cells. Proceedings of the National Academy of Sciences, 2000. 97(16): p. 9042-9046

21. Doisneau-Sixou S et al (2003) Estrogen and antiestrogen regulation of cell cycle progression in breast cancer cells. Endocrine-related Cancer 10(2):179-186

22. Zadra $\mathrm{G}$ et al., Inhibition of de novo lipogenesis targets androgen receptor signaling in castrationresistant prostate cancer. Proceedings of the National Academy of Sciences, 2019. 116(2): p. 631640

23. Little JL et al., Inhibition of Fatty Acid Synthase Induces Endoplasmic Reticulum Stress in Tumor Cells. 2007. 67(3): p. 1262-1269

24. Viswanadhapalli S et al (2019) Estrogen receptor coregulator binding modulator (ERX-11) enhances the activity of CDK4/6 inhibitors against estrogen receptor-positive breast cancers. Breast Cancer Res 21(1):1-15

25. Chen CL et al (2013) Single-cell analysis of circulating tumor cells identifies cumulative expression patterns of EMT-related genes in metastatic prostate cancer. Prostate 73(8):813-826

26. Brigidi GS, Bamji SX, Detection of protein palmitoylation in cultured hippocampal neurons by immunoprecipitation and acyl-biotin exchange (ABE). JoVE (Journal of Visualized Experiments), 2013(72): p. e50031

27. Fan $P$ et al (2007) Long-term treatment with tamoxifen facilitates translocation of estrogen receptor a out of the nucleus and enhances its interaction with EGFR in MCF-7 breast cancer cells. Cancer research 67(3):1352-1360

28. Steinman RM et al (1983) Endocytosis and the recycling of plasma membrane. J Cell Biol 96(1):127

29. Fu S et al (2011) Aberrant lipid metabolism disrupts calcium homeostasis causing liver endoplasmic reticulum stress in obesity. Nature 473(7348):528-531

30. Gong C et al (2020) Phosphorylation independent elF4E translational reprogramming of selective mRNAs determines tamoxifen resistance in breast cancer. Oncogene 39(15):3206-3217 
31. Geter PA et al (2017) Hyperactive mTOR and MNK1 phosphorylation of elF4E confer tamoxifen resistance and estrogen independence through selective mRNA translation reprogramming. Genes Dev 31(22):2235-2249

32. Bollu LR et al (2015) Intracellular activation of EGFR by fatty acid synthase dependent palmitoylation. Oncotarget 6(33):34992

33. Röhrig F, Schulze A (2016) The multifaceted roles of fatty acid synthesis in cancer. Nat Rev Cancer 16(11):732

34. Lee $\mathrm{G}$ et al (2017) Post-transcriptional Regulation of De Novo Lipogenesis by mTORC1-S6K1-SRPK2 Signaling. Cell 171(7):1545-1558.e18

35. Li L et al (2014) Epigenetic identification of receptor tyrosine kinase-like orphan receptor 2 as a functional tumor suppressor inhibiting $\beta$-catenin and AKT signaling but frequently methylated in common carcinomas. Cell Mol Life Sci 71(11):2179-2192

36. Li R et al (2019) ROR2 induces cell apoptosis via activating IRE1 a/JNK/CHOP pathway in high-grade serous ovarian carcinoma in vitro and in vivo. Journal of Translational Medicine 17(1):1-17

37. Fagan DH et al (2017) Acquired tamoxifen resistance in MCF-7 breast cancer cells requires hyperactivation of elF4F-mediated translation. Horm Cancer 8(4):219-229

\section{Figures}


Fig. 1

A.
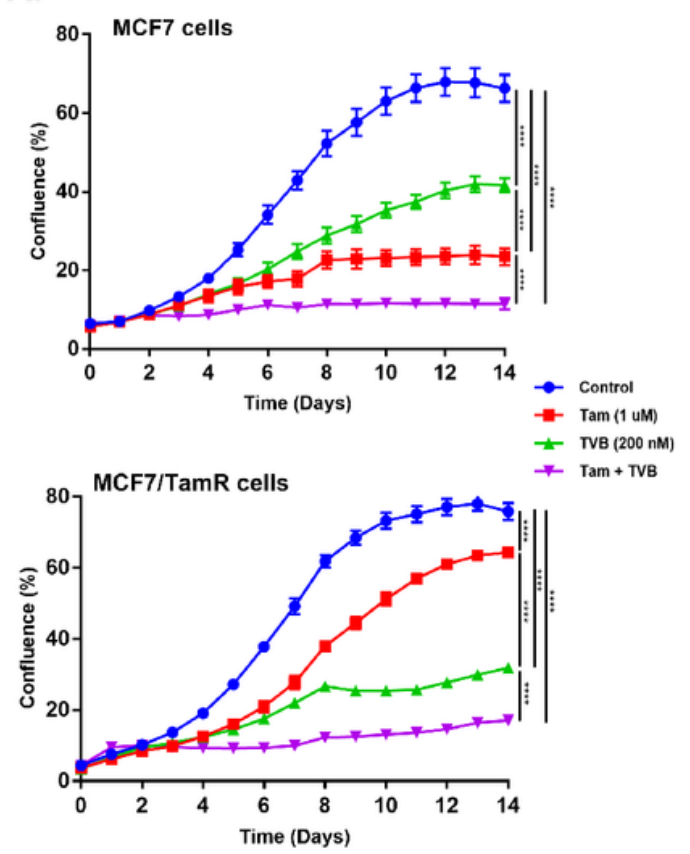

B.
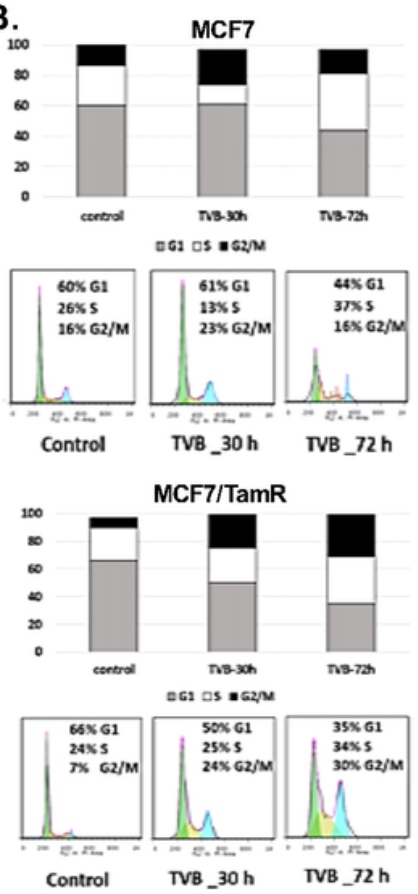

C.
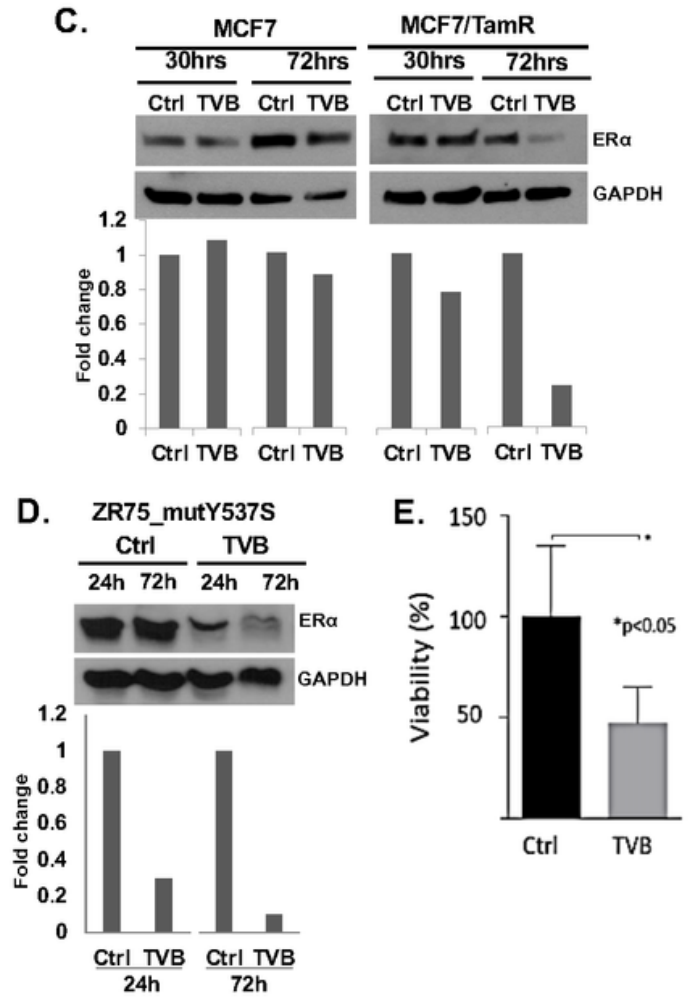

Figure 1

Therapy resistant breast cancer cells have greater sensitivity to FASN inhibition than parental breast cancer cells. a Kinetics of cell growth of either MCF7 (upper) or tamoxifen resistant MCF7 (lower) cells when exposed to vehicle control (blue), $1 \mu \mathrm{M}$ Tamoxifen (Tam) (red), $200 \mathrm{nM}$ of TVB-3166 (green), or both (purple). ${ }^{\star \star \star \star} p<0.0001$. b Comparison of log-scale histograms of propidium iodide-stained cells. Controls were untreated cells. c Western blot analysis of ERa from parental and Tamoxifen resistant MCF7 cells after 30 and 72 hours of exposure to 200 nM of TVB-3166 compared to the control. $d$ Western blot analysis of ERa from estrogen receptor 1 (ESR1) mutant ZR75 cells after 30 and 72 hours of exposure to $200 \mathrm{nM}$ of TVB-3166 compared to control. E) Viability of aromatase inhibitor resistant circulating tumor cells (CTCs) cultured and treated with 200 nM of TVB-3166 
Fig. 2
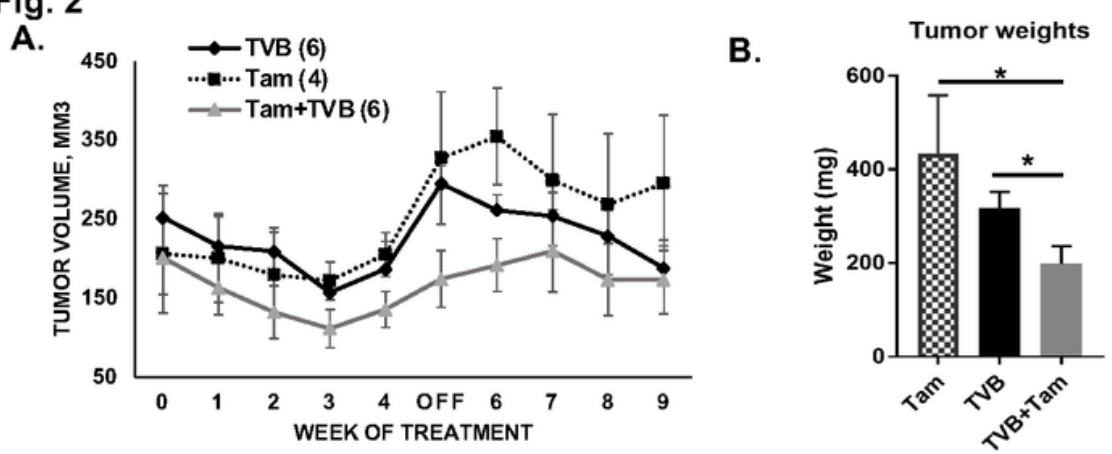

C.

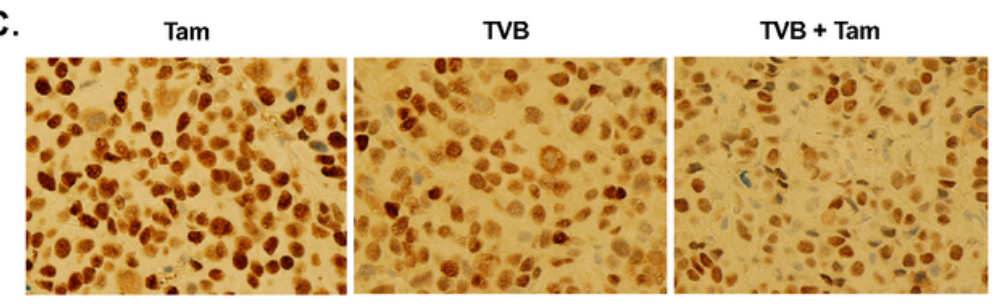

D.

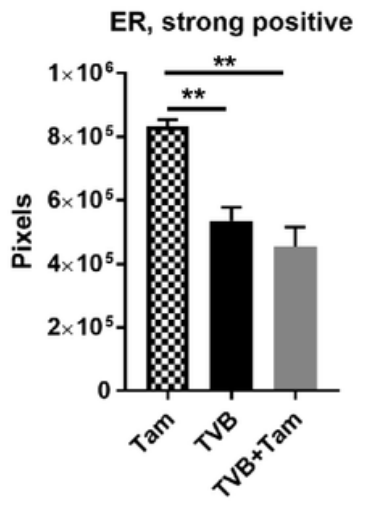

Figure 2

TVB-3166 inhibits tumor growth of tamoxifen-resistant MCF7 xenografts. a Tumor growth curve of MCF7/TamR during treatment. b Weights of extirpated tumor xenografts posttreatment. Tam vs TVB + Tam, $p$ value $=0.047 ;$ TVB vs TVB + Tam, $p$ value $=0.038$. $\mathrm{c} \mathrm{Immunohistochemistry} \mathrm{analysis} \mathrm{of} \mathrm{tumors}$ shows significant decrease in ERa after TVB-3166 treatment alone ( $p$ value $=0.002)$ or in combination with tamoxifen $(p$ value $=0.003)$. $d$ Quantification of data in $c$ 
Fig. 3
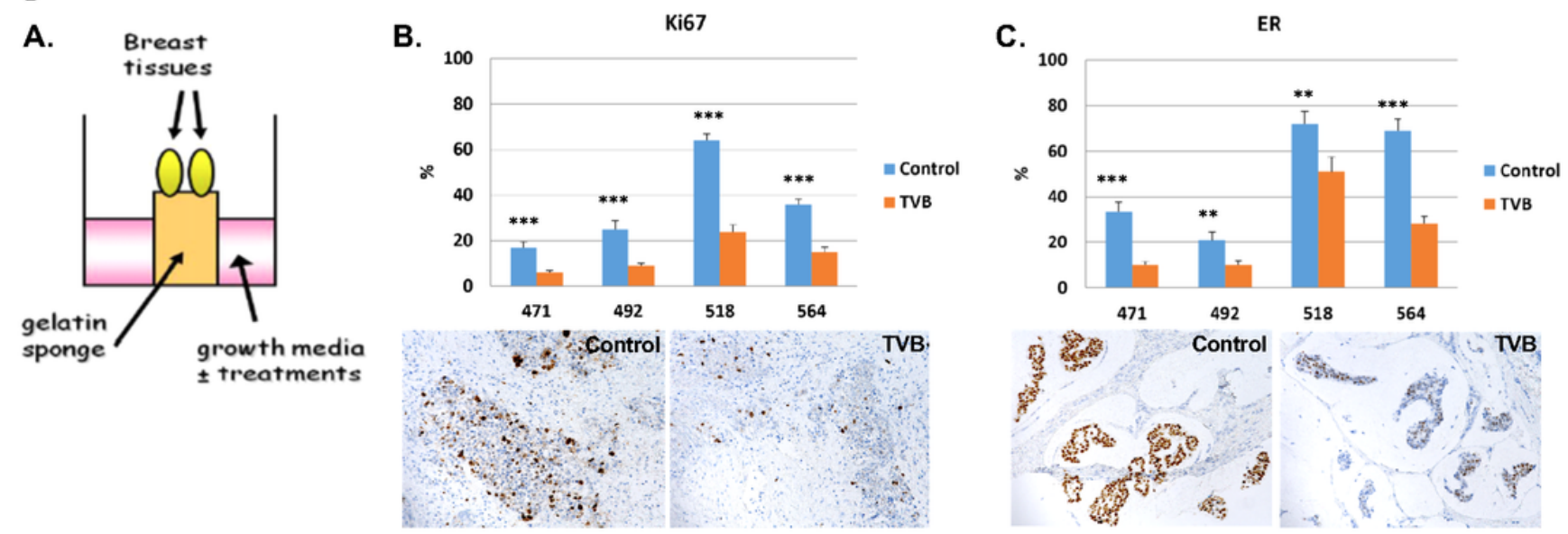

\section{Figure 3}

TVB-3166 inhibits proliferation and reduces ERa expression in patient derived primary tumor explants. a Schematic representation of ex vivo culture model. b Immunohistochemistry analysis shows that TVB3166 treatment leads to a decrease in proliferation, demonstrated by a reduction in Ki67 expression. c Immunohistochemistry shows a reduction in ERa expression upon treatment with TVB-3166 of the patient derived primary tumor explants 
Fig. 4

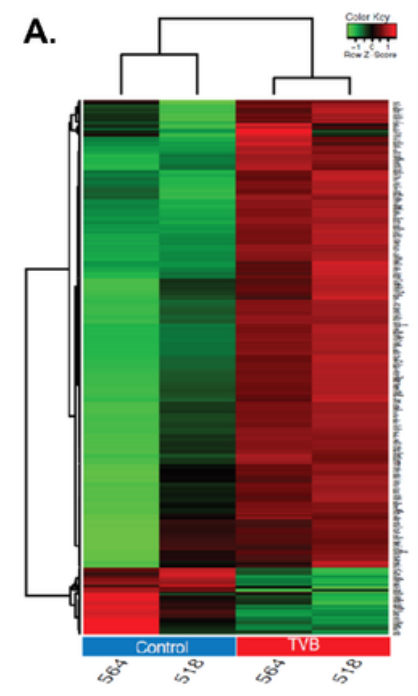

B.

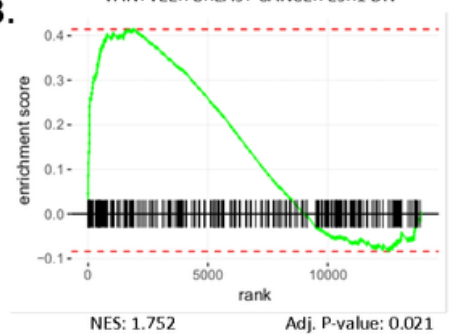

C.

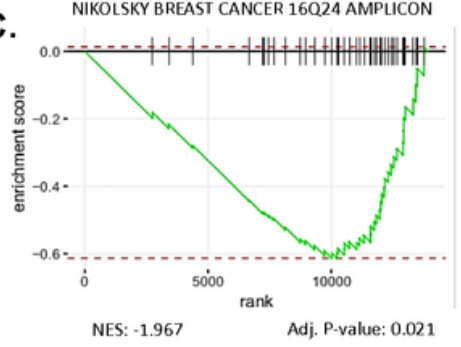

D.

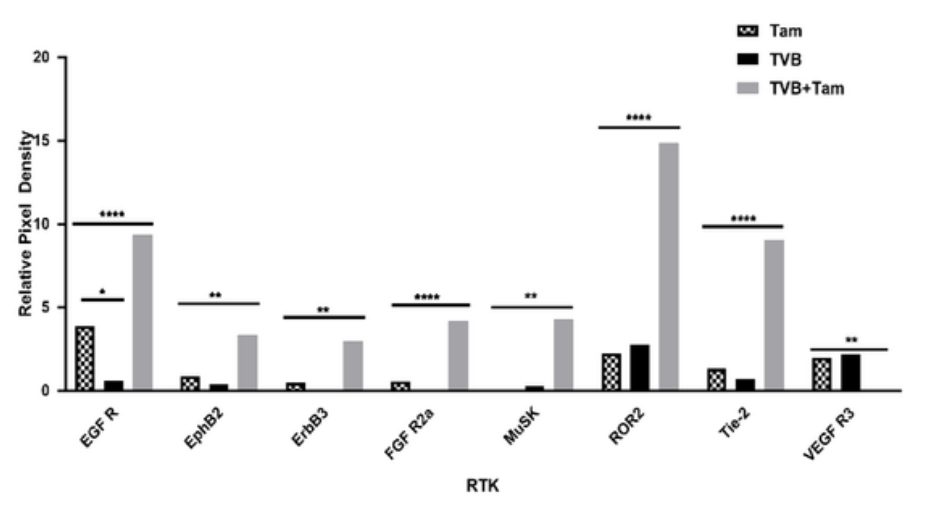

\section{Figure 4}

Identification of differentially expressed genes between vehicle-treated and TVB-3166-treated patientderived primary tumor explants, 564 and 518 . a Heatmap representing differentially expressed genes between vehicle and TVB-3166. $b$ and $c$ Representative gene set enrichment analysis (GSEA) plots showing 2 enriched gene sets: genes downregulated in ESR1 positive breast tumors compared to ESR1 negative tumors (positive correlation) (b) and invasiveness signature resulting from cancer cellmicroenvironment interaction (negative correlation) (c). $d$ Representative bar graph of phospho-Receptor Tyrosine Kinase (RTK) assay showing statistically significant changes in protein phosphorylation in MCF7/TamR cells treated with $200 \mathrm{nM}$ of TVB-3166, $1 \mu$ M Tamoxifen, or both. Phosphorylation of ROR2 and EGFR were the most increased in response to combination treatment. $* \star \star \star ~ p<0.0001 ; * \star * p<0.0005$; ${ }^{* *} p<0.005 ;{ }^{*} p<0.05$ 
Fig. 5

A.
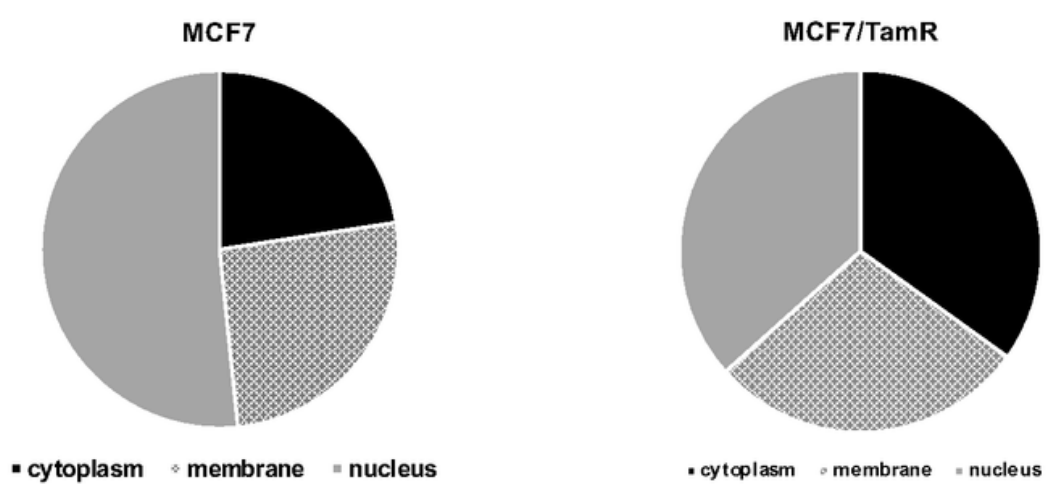

B.

ER $\alpha$ degradation after TVB

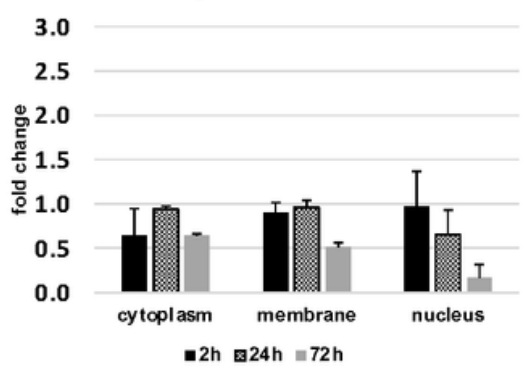

ER $\alpha$ degradation after TAM

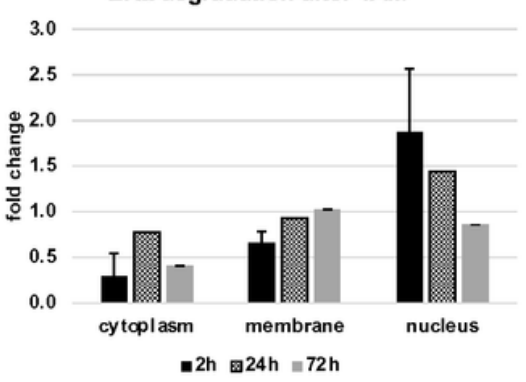

ER $\alpha$ degradation after TVB+TAM

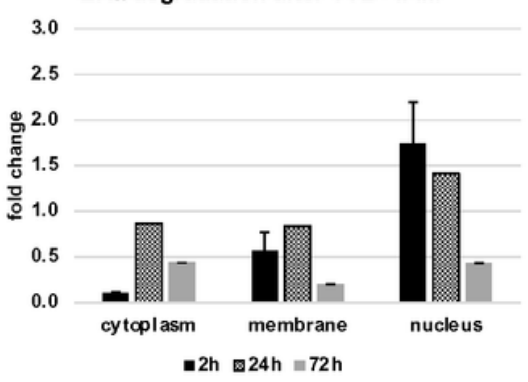

\section{Figure 5}

TVB-3166 alters subcellular localization of ERa and induces its degradation in tamoxifen resistant breast cancer. a The pie charts show subcellular localization of ERa in MCF7 and MCF7/TamR cells determined by western blotting. ERa expression in the cytoplasm is significantly more in MCF7/TamR cells compared to parental MCF7 cells ( $p$ value $=0.025$ ). b Bar graphs represent degradation of ERa protein in MCF7/TamR cells determined by western blotting. TVB-3166 and Tamoxifen combination treatment leads to increased ERa degradation 
Fig. 6

A.

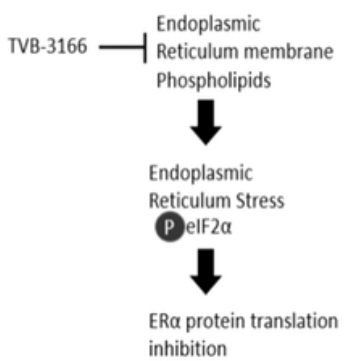

C.

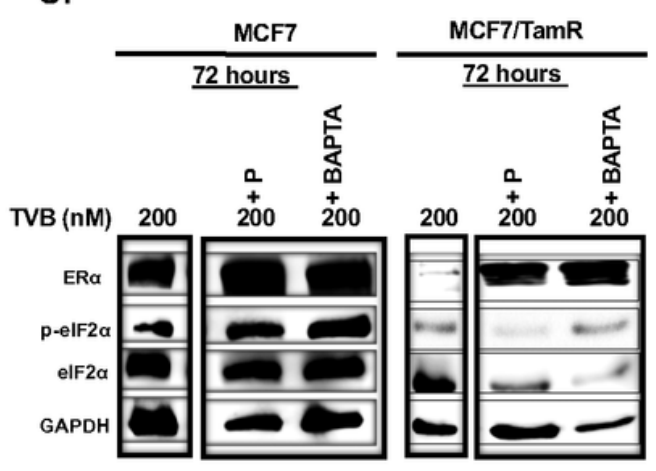

B.

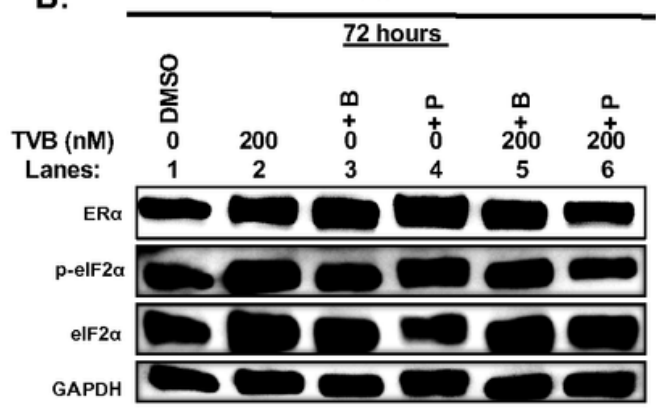

MCF7

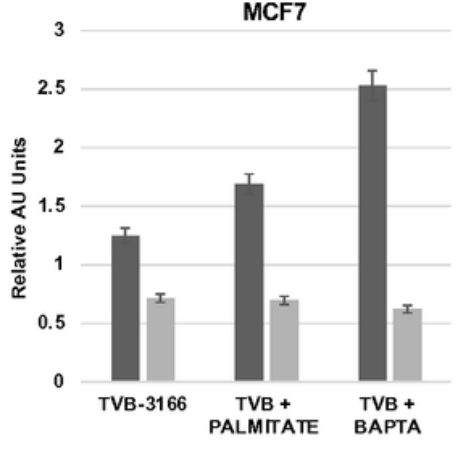

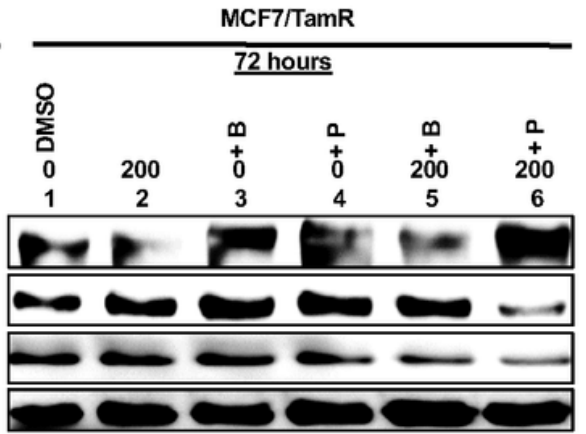

MCF7/TamR

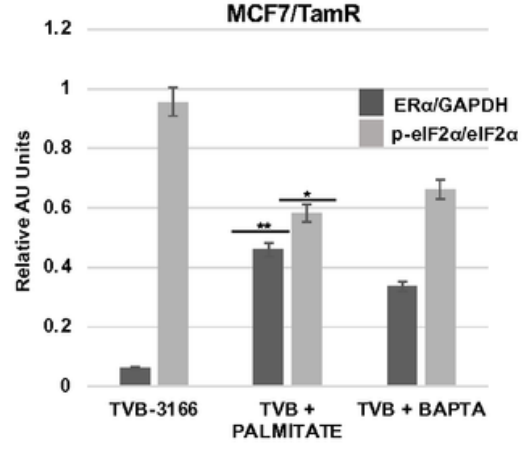

Figure 6

TVB-3166 induces endoplasmic reticulum stress (EnRS) that targets ERa in tamoxifen resistant breast cancer. a Proposed model of TVB-3166 induced ERa degradation in tamoxifen resistant breast cancer. $b$ Western blot analysis of MCF7 and MCF7/TamR cells treated with 200 nM TVB-3166 for 72 hours, or TVB-3166 with either palmitate $(P)$ or BSA (B). Palmitate was conjugated to BSA in a 6:1 molar ratio. Bands were quantified using area under the curve analysis with NIH ImageJ software. Graphs represent an average of 3 independent experiments. ERa was standardized to GAPDH, while p-elF2a was standardized to total elF2a. ** indicates a statistical change in ERa/GAPDH between TVB-3166 and TVB + PALMITATE treated group $(p=0.02)$ * indicates statistical change of $p$-elF2a/elF2a between TVB-3166 and TVB-3166 + PALMITATE group $(\mathrm{p}=0.05)$. c Western blot analysis of MCF7 and MCF7/TamR cells. Both cell lines were pretreated with EnRS inhibitor, BAPTA, for 60 minutes prior to treatment with TVB3166 in order to compare to the effects of TVB + PALMITATE

\section{Supplementary Files}


This is a list of supplementary files associated with this preprint. Click to download.

- Figs1.png

- Figs2.png 